\title{
Geomagnetic Reversals in Quaternary Revealed from a Palaeomagnetic Investigation of Sedimentary Rocks
}

\author{
Vaclav Bucha \\ Geophysical Institute of Czechoslovak Academy of Sciences Prague
}

(Received February 3, 1970)

\begin{abstract}
:
Eight cycles of sedimentary rocks covering approximately the time interval of nearly one million years were investigated by palaeomagnetic methods. The reversal of the field from the reversed to the normal polarity was found at approximately 950,000 years ago and the detailed course of direction and intensity changes of the geomagnetic field is described. Short temporal field reversals occurred according to our dates even in younger periods. The results offer a new correlation method for geology.
\end{abstract}

\section{Introduction :}

In some well-preserved sedimentary areas in Czechoslovakia, regularly alternating loess, water sediments and forest soils were found which form terraces of old river beds.

The origin of the individual rock types seems to depend on the climatic changes between cold and warm periods and on their periodical repetition in seasonal insolation change. At the locality of Cerveny kopec near Brno eight sedimentary cycles on the whole were discovered below the Holocene, each of which had been developed for 115,000-127,000 years, according to isotope dating and to the astronomical curve of winter insolation at $55^{\circ} \mathrm{N}$ latitude which was constructed for this purpose (in it the so-called "normal cycle" of 115,000-117,000 years and the "long cycle" of 125,000-127,000 years duration have been distinguished by Kukla (1968). The cycles mentioned cover the time interval of nearly one million years and are indicated as $B$ to $I$. Cycle $B$ very probably lasted between 10,500 and 128,000 years, whereas, e.g. cycle I approximately covers a time interval of 845,000 to 962,000 years. The age determined may obviously show deviations of some thousands of years but, in spite of that, the mighty sedimentary formations represent very interesting material for the chronological study of magnetic properties and for the investigation of new laws of earth's magnetic changes. Cycle $I$ on Cerveny kopec near Brno, geologically well elaborated, is approximately $4 \mathrm{~m}$ thick, so that, assuming the same velocity of sedimentation, a layer of $2 \mathrm{~cm}$ thickness was deposited over a period of 600 years. From the point of view of the considerably varying geomagnetic secular variation, these sedimentary rocks represent, in comparison to Quaternary volcanic rocks, much more comprehensive material, the paleomagnetic investigation of which could, under favorable circumstances, contribute significantly to explaining the dynamics of the earth's magnetic field 
in the past.

The basic question was to examine if the rock samples mentioned have remanent magnetization and what are its characteristics. Measurements of some samples of loess and soils on a Jelinek spinner magnetometer (sensitivity $4 \times 10^{-9}$ units CGSM) (Jelinek, 1966) showed that the value of remanent magnetization is between $10^{-5}$ to $10^{-7} \mathrm{CGSM}$, certain parts being viscous magnetization. At the same time we found that some samples of cycle $I$ showed reversed magnetization which offers the possibility of investigating the mechanism of polarity reversals of the earth's magnetic field.

1. Character of the geomagnetic reversal of 950,000 years ago

More than 200 regular oriented samples (cubes with edges of $2 \mathrm{~cm}$ ) were collected from a wall, $4 \mathrm{~m}$ thick, of cycles $I$ and $H$ (962,000-825,000 years) and set in the laboratory in the same position with respect to the earth's magnetic field as they had been collected from the locality, for one week. In addition to these, in each case 5 samples were collected from seven approximately equally distant points of different heights along the whole wall and these were also used for laboratory investigations.
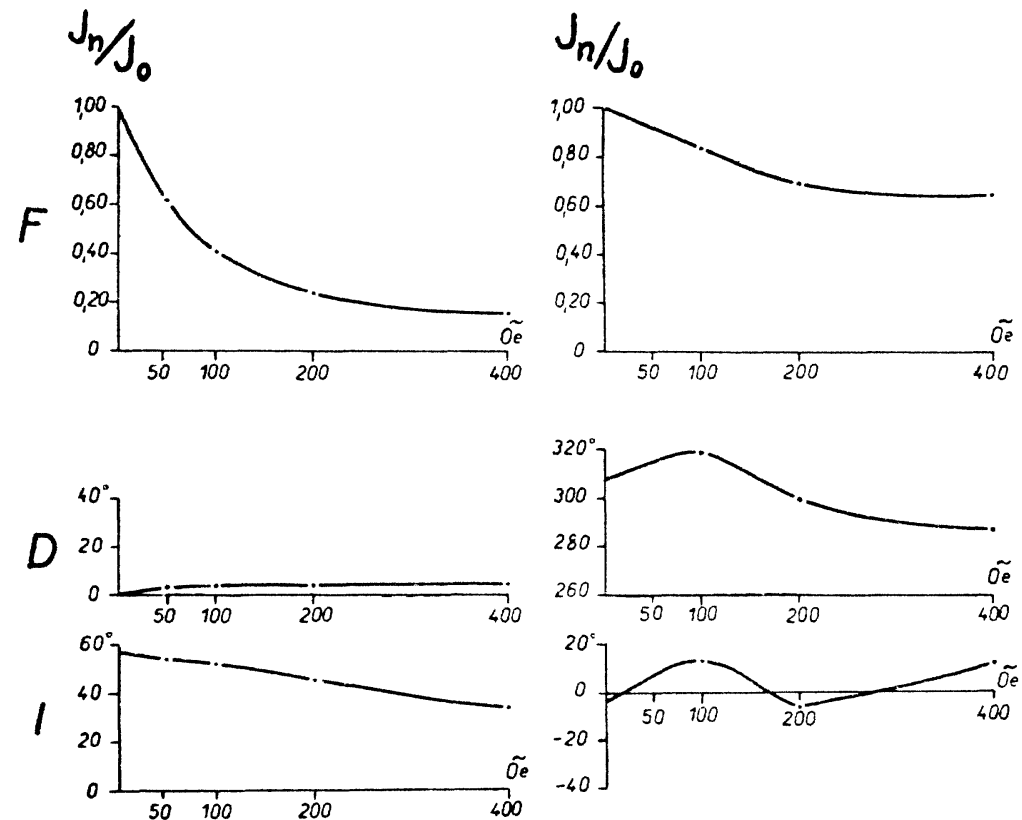

Fig. 1a Changes F,D,I during A.C. demagnetization of the sedimentary rock sample No. 1a that was set in the laboratory in the same position with respect to the earth's magnetic field as it had been collected from the locality, for one week; remanent magnetization of the sample was of normal polarity

Fig. 1b Changes F,D,I during A.C. demagnetization of the sample No. $1 b$ (from the same layer as was sample 1a) with normal polarity of the remanent magnetization. Before the procedure, the sample was left for one week in a position turned by $180^{\circ}$ around the E-W axis (i. e. against the direction of the earth's magnetic field) 


\section{a) Magnetic cleaning by A.C. field and heating.}

First, after one week, these samples were demagnetized by an A.C. field and heated. After A.C. demagnetization in a 50 Oe field the remanent magnetization of the normally magnetized samples already showed an intense decrease (Fig. 1a), while the remanent magnetization of the reversely magnetized samples increased considerably in comparison to their original values (Fig. 2a.). The sample which had a positive dip before the A.C. demagnetization but a negative one after demagnetization, at first showed a decrease of magnetization when the A.C. field of 50 Oe was applied (Fig. 3a). The remanent magnetization increased to maximum when an A.C. field of 100 Oe was applied. In higher fields, the remanent magnetization decreased again. All these changes proved the presence of a relatively strong component of viscous (unstable) magnetization, originating in the rocks due to the long-termed action of the earth's magnetic field. As can be seen from Figs. 1a, $2 \mathrm{a}, 3 \mathrm{a}$, the rapid changes of the remanent magnetization are accompanied by changes of the declination and the dip if the samples are demagnetized in an A.C. field of 100-200 Oe. When higher values of the A.C. field were applied, the changes of $D$ and $I$ were small; that means A.C. demagnetization at 100-200 Oe makes it possible to clean the unstable components of remanent magnetization magnetically.

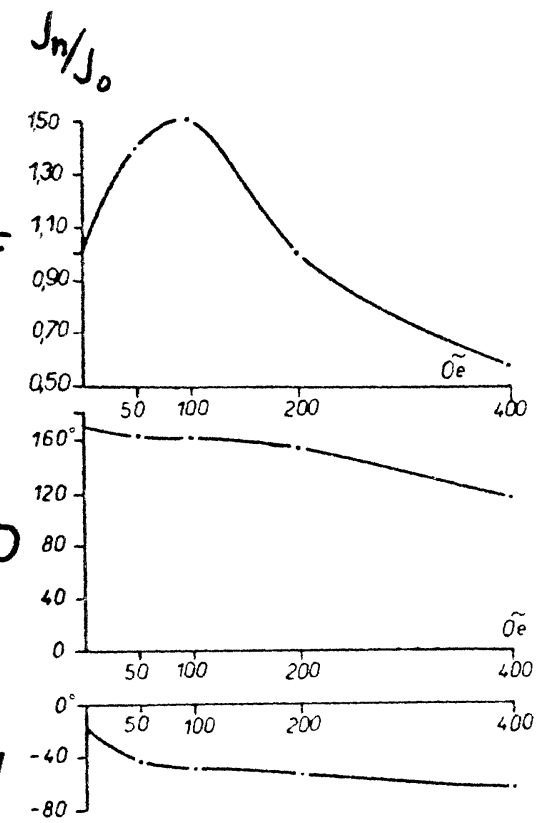

Fig. 2a Changes F,D,I during A.C. demagnetization of the sample No. 2a (set in its original position for one week) with original negative polarity of remanent magnetization (negative inclination I)

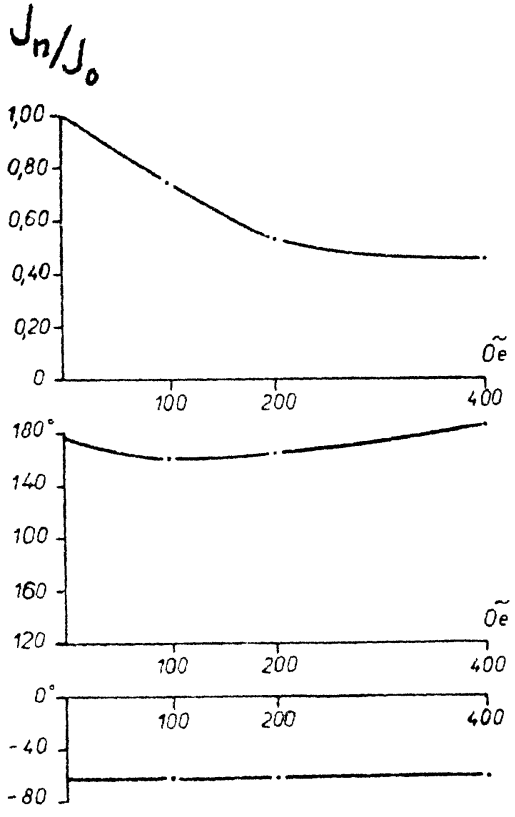

Fig. 2b Changes F,D,I during A.C. demagnetization of the sample No. $2 b$ (from the same layer as was sample 2a) with negative polarity. It was turned by $180^{\circ}$ around E-W axis and left for one week before the procedure in the opposite position (against the present-day field) than it was at the locality 
In the same way thermal magnetic cleaning was applied to some typical samples. Expressive changes in the direction of magnetization (see Fig. 4a, 5a) were found when temperatures increased. After applying higher temperatures, however, some distortion of direction took place, because even small uncompensated magnetic fields in the Helmholtz coils, in which the non-magnetic furnace was placed, can create new disturbing components of remanent magnetization.

The representative samples were then left for one week in a position turned by $180^{\circ}$ around the $E$ - $W$ axis (e. g. against the direction of the earth's magnetic field) and then they were magnetically cleaned. The results of the A.C. demagnetization of these samples show a very slow and regular decrease of the remanent magnetization (Fig. 1b, $2 \mathrm{~b}$, $3 \mathrm{~b}$ ) and only small changes of $D$ and $I$ during the cleaning. The thermal magnetic cleaning also shows only small changes in the directions of the remanent magnetization (Fig. $4 \mathrm{~b}, 5 \mathrm{~b}$ ) although, due to the disturbing effects mentioned, its directions change somewhat.

The magnetic cleaning showed that the samples of sedimentary rocks, taken on the defile, contain three types of magnetizations : primary stable magnetization which origin-

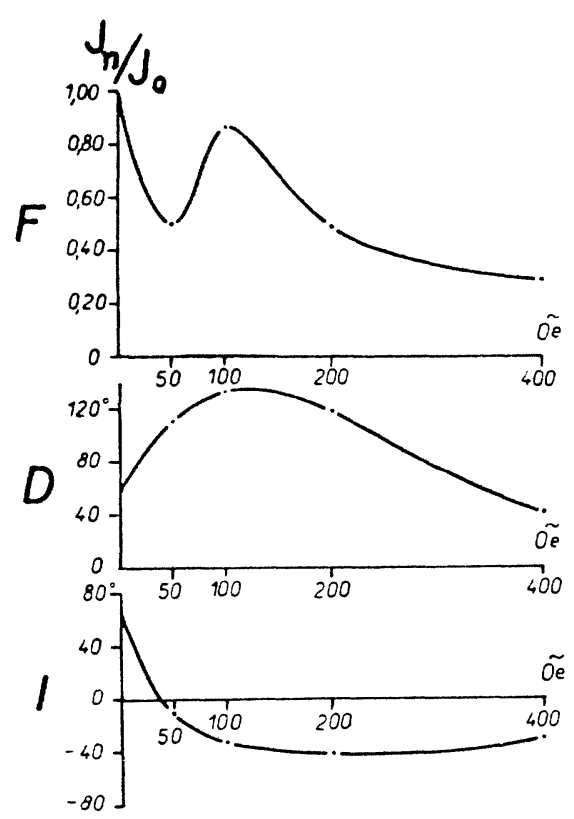

Fig. 3a Changes F,D,I during A.C. demagnetization of the sample 3a (left for one week in its original position; (its inclination was positive at first and during demagnetization it changed into the negative one)

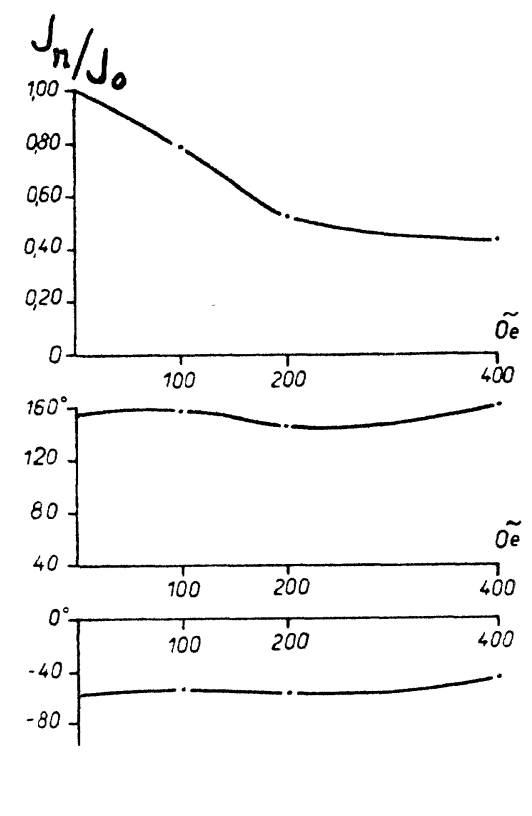

Fig. 3b Changes F,D,I during A.C. demagnetization for the sample No. $3 b$ (from the same layer as was sample 3 a) set before the procedure in the reversed position. The acting of the geomagnetic field on the sample during one week caused that its inclination has changed to $-58^{\circ}$ which did not change during demagnetization 
ated during the sedimentation of the rock; viscous unstable magnetization which disappears from the samples within some days after being deposited in a reverse position to that in which they were on location; secondary metastable magnetization, approximately equal in value to the unstable magnetization. This third type of magnetization probably originated in the rock as a result of chemical processes during the existence of the rock. If the samples were only to contain stable and unstable magnetizations, expressive changes would become apparent in the direction of magnetization when the samples were placed in the reversed position for one week. The demagnetizing curves obtained prove quite unambiguously that the unstable magnetization newly originated as a result of the samples being in the reversed position with respect to the earth's magnetic field, compensates the metastable magnetization so that only the stable magnetization remains in the sample. This conclusion may clearly be seen in Figs. $2 b, 3 b$, where the direction of remanent magnetization remains practically unchanged during A.C. demagnetization. This simple procedure of letting the earth's magnetic field act on samples in their reversed position for a time, enables us to obtain easily the direction and values of the remanent magnetization for larger number of samples with relatively high accuracy, as was proved above; a check by means of A.C. cleaning for selected samples is naturally desirable.
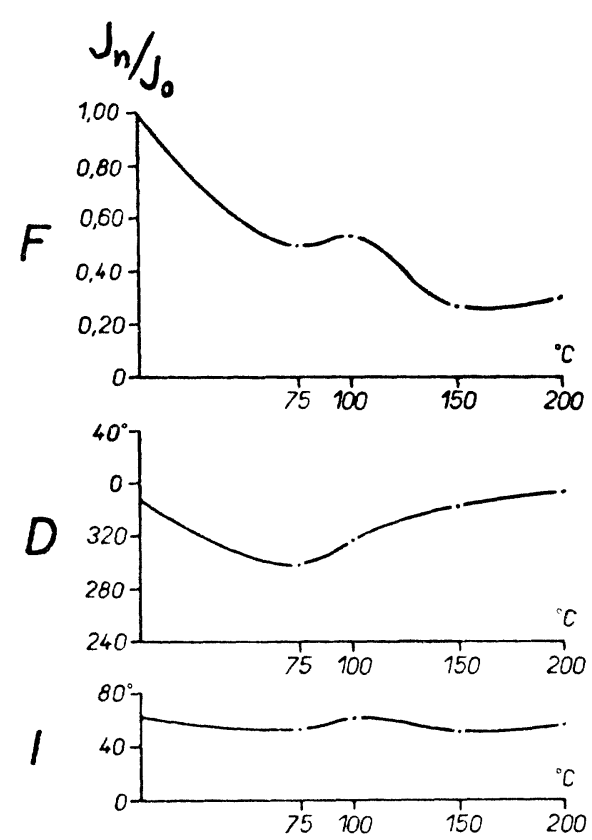

Fig. 4a Changes F,D,I during the temperature magnetic cleaning of the sample No. 1c with normal polarity of remanent magnetization which was left in its original position for one week

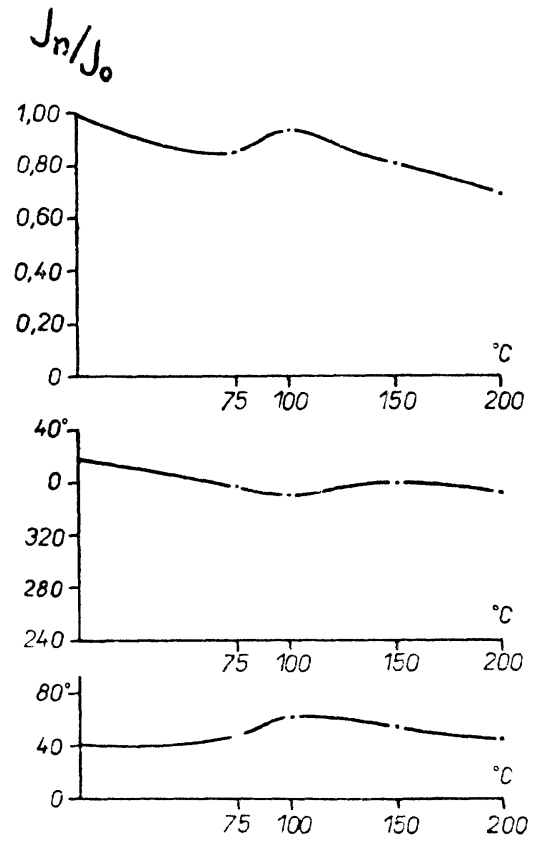

Fig. 4b Changes F,D, I during the temperature magnetic cleaning of the sample No. 1e (from the same layer as was sample 1c) with normal polarity (set in the opposite position before procedure for one week) 

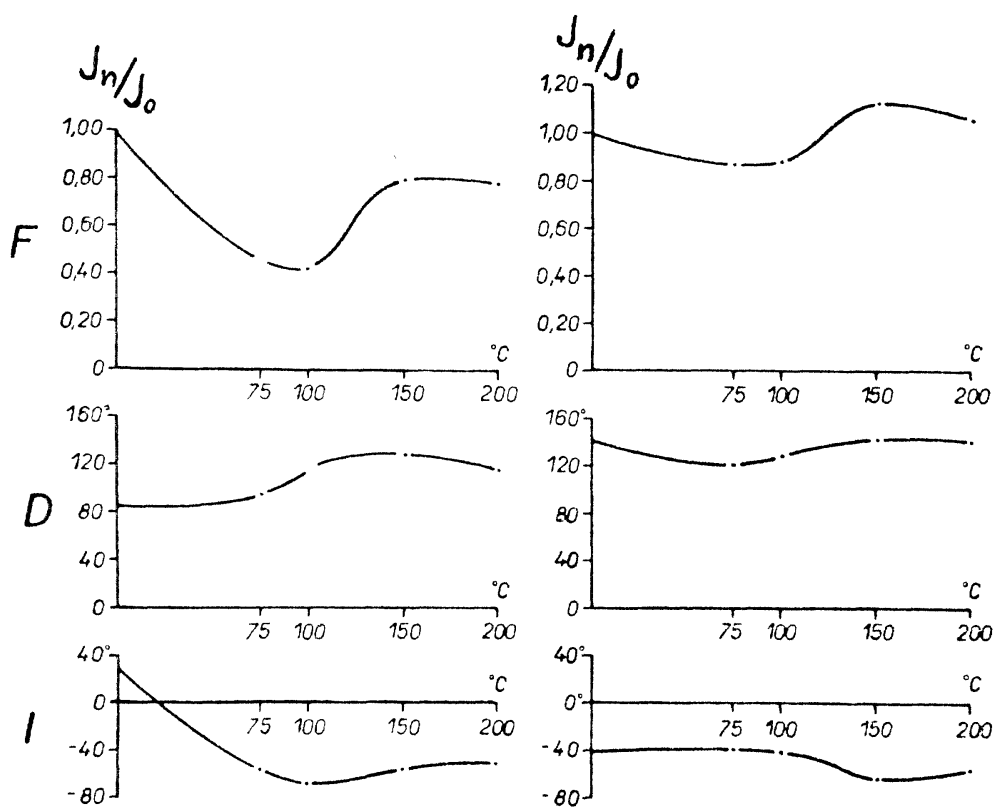

Fig. 5a Changes F,D,I during the temperature magnetic cleaning of the sample No. 3c which has been left in its original position as at the locality for one week; the inclination displayd changes during the process of demagnetization from positive values to the negative ones

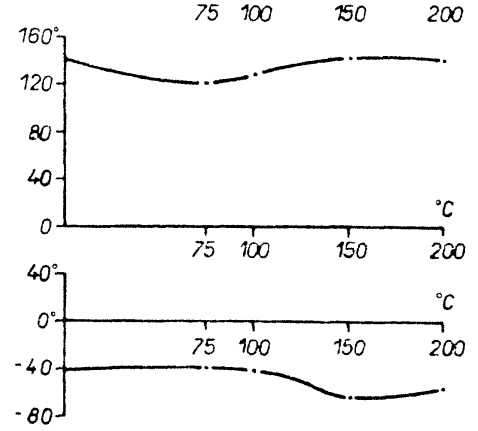

Fig. 5b Changes F,D,I during the temperature cleaning of the sample No. $3 d$ left in the opposite position for one week; during this time the inclination changed from the positive values to the negative ones so that in the course of demagnetization the dip changes were very small.

\section{b) Changes of the geomagnetic field direction during the polarity reversal}

The investigation of a collection of 200 samples, taken successively along a defile, $4 \mathrm{~m}$ thick, from sedimentary cycle $I(962,000-825,000$ years), was carried out by means of this method. Due to a certain dip of the sedimentary layers, the 5 samples taken from the same height at seven places of the defile for laboratory experiments do not represent exactly the same age, especially when considering that every sample on the average covers a sedimentation interval of 600 years. Therefore, the direction as well as the value of adjacent samples may differ mutually somewhat. The time dependence of the remanent magnetization, the declination and the inclination for cycle $I$ is given in Figs. 6, 7, 8. The upper curves represent the changes before cleaning (obtained by measuring samples placed for a week in the position they were before), while the lower curves characterize the values after magnetic cleaning (obtained by measuring samples placed in the reversed position with respect to the earth's magnetic field). For selected samples a check of the values was carried out by laboratory cleaning. These curves indicate that the sedimentation of the samples examined took place during the reversal of the earth's magnetic field. 


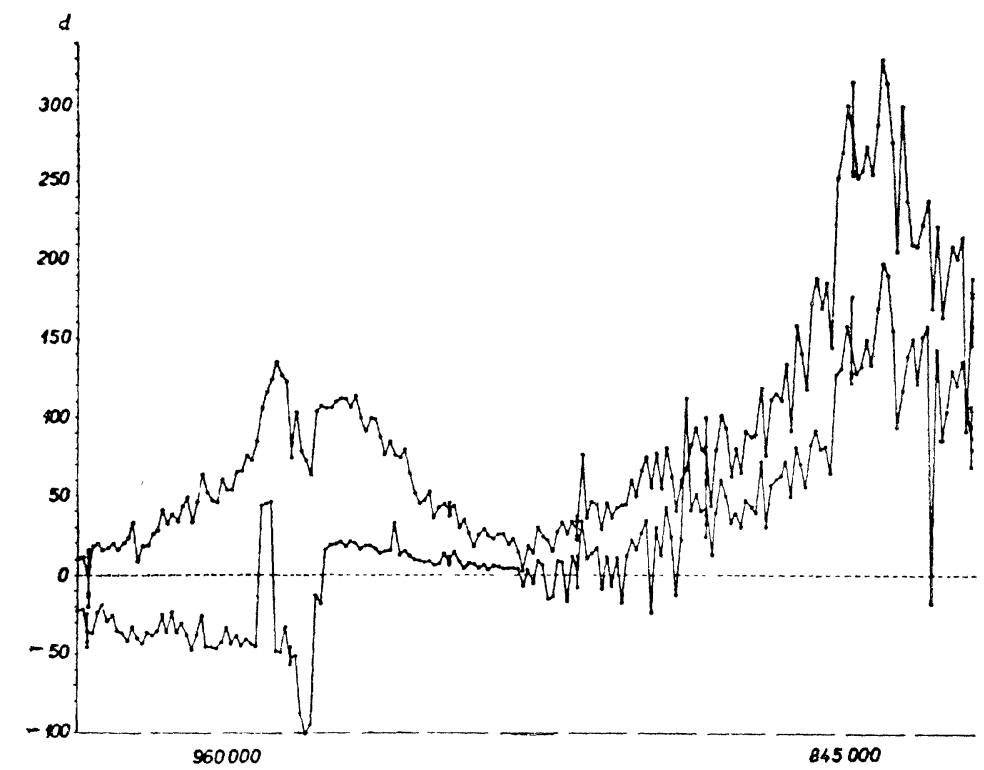

Fig. 6 Temporal dependence of the remanent magnetization $\mathrm{J}$ of 200 samples for sedimentary cycles I and $\mathrm{H}(962,000-825,000$ years). The upper curve represents the course before magnetic cleaning, i.e. it includes the stable, unstable, and metastable magnetization. The points representing the stable "cleaned" magnetization (lower curve) are drawn in such a way that the values with negative dip are considered to be negative and are sketched below the $\mathrm{x}$-axis.

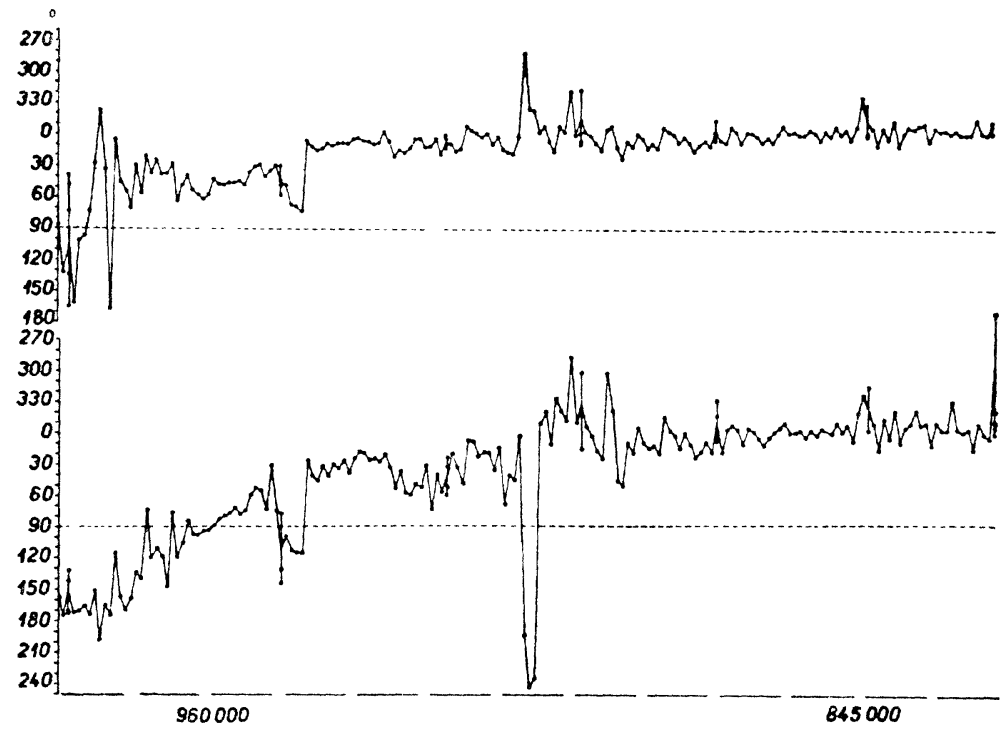

Fig. 7 Temporal dependence of the changes in magnetic declination for the sedimentary cycles $I$ and $H$. The upper curve represents the course of declination before magnetic cleaning of samples, the lower curve is valid for the magnetically cleaned values $D$ 


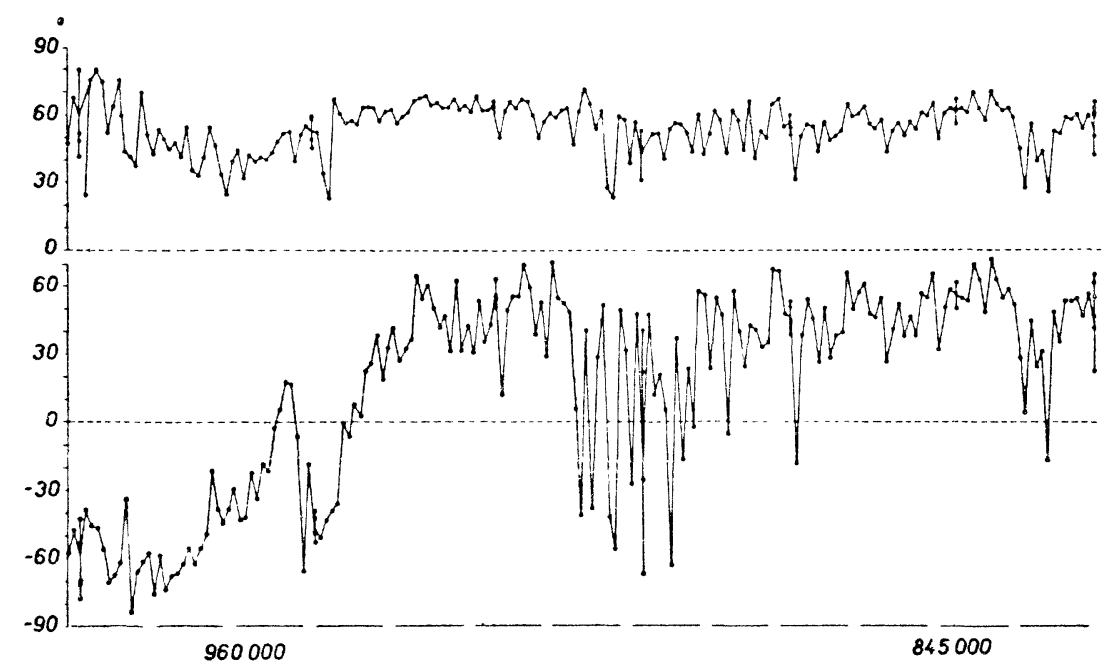

Fig. 8 Temporal dependence of the changes in magnetic inclination (see text in Figs. 6, 7)

Beginning of the lower curves for $D$ and $I$ after cleaning corresponds to values which characterize the reversed direction of the field in comparison with the present-day field. Even if we admit some inaccuracy in determining the time by means of the insolation curve, it is clear that the reversal of the field from the opposite to the normal polarity took place approximately 950,000 years ago. According to Cox (1968), this is in good agreement with his reversed-to-normal change of polarity called jaramillo event. The prevailing normal polarity found for younger periods would not prove, however, the youngest, reversed part of the Matuyama epoch. To verify, if this reversal applies to 950,000 or 700,000 years (Matuyama-Brunhes reversal), more measurements are necessary.

The whole process set in quite rapidly, at first, for a shorter time. It was then followed by repeated returning to the reversed values for a short time, but then again the normal polarity of the field was established. After a certain time (approximately 30,000 years) an intermediate phase appeared which was defined by alternating positive and negative values of inclination and by declination, differing by $180^{\circ}$. After that the field became calmer and $D$ and $I$ approximated to values, characterizing the direction of the presentday earth's magnetic field.

Expressive oscillations with shorter periods represent an interesting feature. Their only explanation may be in the considerable dynamics of the geomagnetic changes. The composition of the sedimentary rocks investigated changes along the defile very slowly so that the magnetic properties of neighbouring samples cannot differ too much. The strongly alternating amplitudes of the oscillations are very interesting; whereas in the youngest section of the curves they were marked, they are absent in the section immediately following the reversal. This phenomenon may be explained either by big differences in the rate of sedimentation or by different dynamics of the earth's magnetic field within various periods. 
The upper curve for the remanent magnetization (Fig. 6) which characterizes "uncleaned" values, shows two expressive maxima. The smaller one took place during the reversed polarity of the geomagnetic field, the second one appeared in the younger periods when the polarity was normal. There the biggest differences between cleaned and uncleaned curves were found, which manifest a very strong component of the unstable remanent magnetization. The samples showing increased remanent magnetization are darker in colour than other samples. After cleaning the first maximum of remanent magnetization (i.e. during reversed polarity) disappeared, whereas the second one remained. The reason is probably in the fact that the field around 845,000 years ago, in which the samples with higher magnetization originated, must have had a much larger value than during the reversal. More precise answers can be given, however, on the basis of the following experiment.

\section{Geomagnetic intensity and its changes during reversal}

The knowledge of the geomagnetic field intensity and its changes in the past represents an important problem when the mechanism of the reversal of the earth's magnetic field is to be discovered. Considering at least two different types of sediments (light-brown and dark-brown), it is difficult, however, to deduce the values of the geomagnetic intensity for the whole $4 \mathrm{~m}$ thick defile directly from the remanent magnetization.

\section{a) Artificical resedimentation of samples}

It is known that the intensity of the field, acting during rock sedimentation in water, together with the content of ferromagnetic minerals, are the main factors which influenced the value of the remanent magnetization at the time of origin of the sedimentary rocks. Therefore, we carried out experimental artificial sedimentation of samples in different magnetic fields. For these purposes two samples (one dark, one light-brown) were selected, the magnetization of which was known. The artificial resedimentation of a crumbled sample dissolved in water were carried out first in a geomagnetic field equal to the presentday one, in a special device (Fig. 9) in which a sample of the same size as the original cube was left to sediment. After measuring it, the procedure was repeated in a field of double intensity with the same sample. The values of the magnetization were calculated by summing up the measurements of remanent magnetization of the samples placed in the position in which they sedimentated and then in a position reversed by $180^{\circ}$ around the $E \cdot W$ axis for a week. One cannot suppose that the samples could have acquired the metastable component within a few days of their resedimentation. The results of the measurements of artificially sedimented samples brought some interesting conclusions. The light-brown sample, No. 1 (age 900,000 years) yielded an original remanent magnetization of $1.1 \times 10^{-6} \mathrm{CGSM}$, after artificial sedimentation in the present-day field the remanent magnetization was $3.6 \times 10^{-6} \mathrm{CGSM}$ and in the field of double intensity $6.0 \times 10^{-6}$. The dark-brown sample, No. 2 (age 860,000 years), showed an original magnetization of $18.0 \times$ $10^{-6}$ CGSM, after resedimentation in the present-day field 6.1 and in the field of double intenisty $13.2 \times 10^{-6}$ CGSM. It follows, under assumption both primary sedimentation and resedimentation of rock samples were similar processes that the earth's field in which 
sample No. 1 originated in nature was one-third of the present-day field, whereas the sedimentation of sample No. 2 took place in a field which was 2.7 times the present-day field. It is obvious that some other factors could influence the original magnetization (e.g.

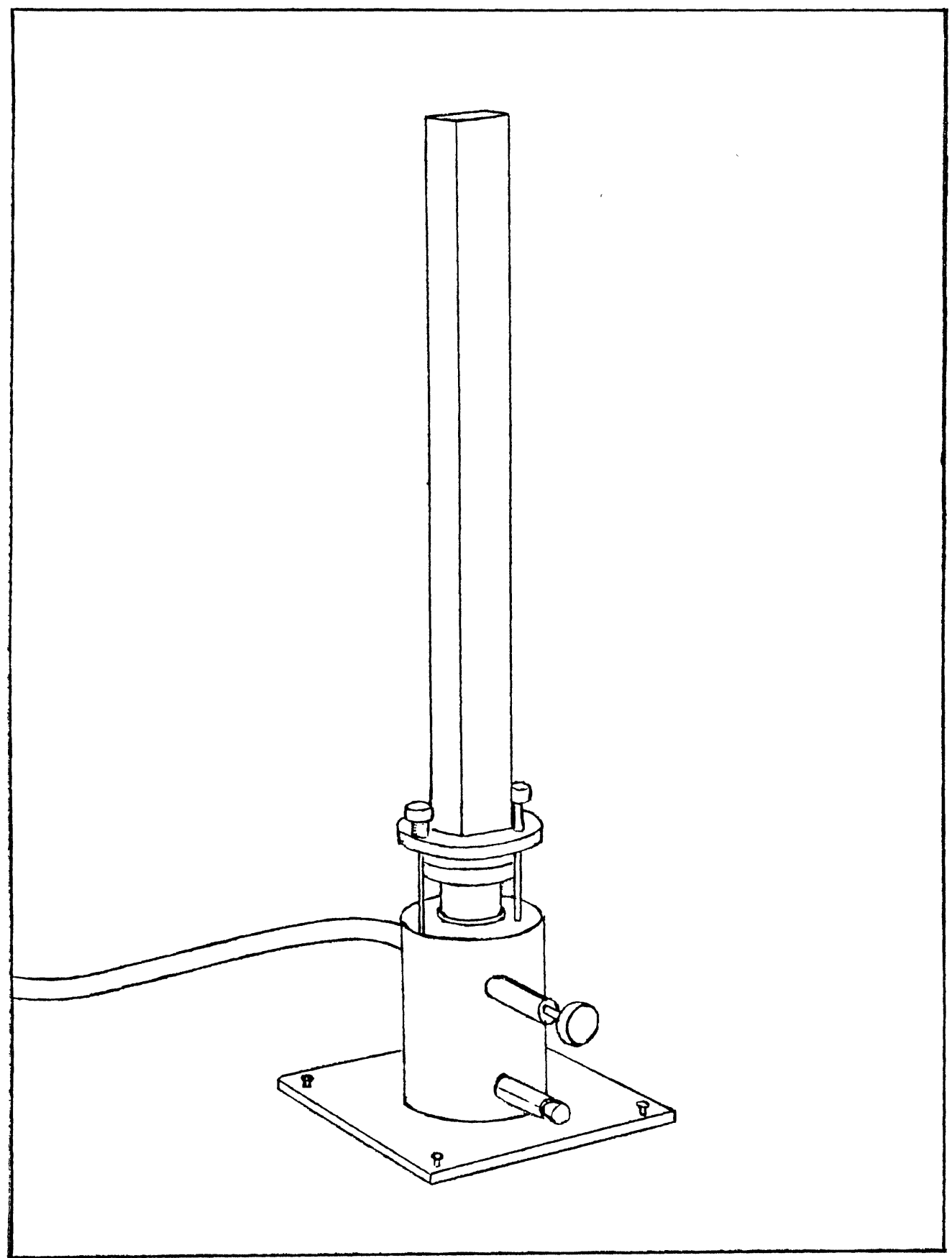

Fig. 9 Device for artificial resedimentation of samples in water in differing magnetic fields 
some chemical changes their effect being difficult to be determined). Considering our archaeomagnetic investigations, which indicate that the field around 400 B.C. was higher than double the present-day field (Bucha. 1967), we may admit similar fluctuation of the earth's magnetic field in the past, too. The presence of various ferromagnetic minerals in both prevailing types of rocks investigated (dark and light-brown) along the whole defile, obviously gave rise to different magnetizations of both samples in an equal field. (The light-brown sample in the present-day field displayed a remanent magnetization of only $3.6 \times 10^{-6}$ CGSM, whereas the darkbrown sample a remanent magnetization of $6.1 \times 10^{-6}$ CGSM). Therefore, we tried to indicate by means of dotted curves 1,2, 3 in Fig. 10, to which field the examined remanent magnetization corresponds (or in other words, what field was required to generate the corresponding magnetization). Curve 1 represents an intensity of 0.475 0e (the present-day field in Prague), curve 2 corresponds to the double that field $(0.950 \mathrm{e})$, curve 3 to $1.4250 \mathrm{e}$. The maxima on the dotted curves indicate the occurrence of dark-brown sedimentary rocks, which acquired a stronger magnetization in the same field than the light-brown sediments.

b) Character of the geomagnetic intensity changes during reversal

The following conclusions concerning the values and changes of the earth's magnetic field can be deduced from the curve for the remanent magnetization of sedimentary rocks along the defile of cycle $I$. At the origin of the curve, where the polarity of the field was reversed, the geomagnetic intensity varied between its present-day value and 1.5 times

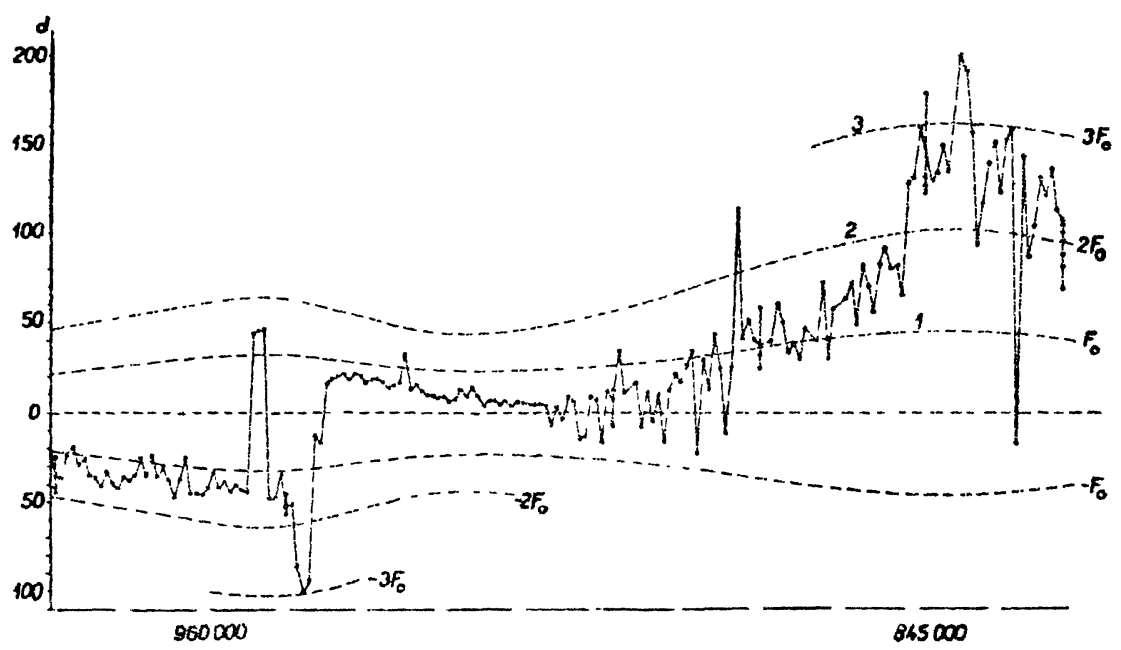

Fig. 10 Changes of the earth's magnetic intensity $\mathrm{F}$ given by the curve for the stable remagnetization and by curves 1, 2, 3 for the geomagnetic field determined from measurements of the artificially sedimented samples, gradually in the present-day field (curve 1), in double its value (curve 2) and in triple its value (curve 3). Maximum values of curves $1,2,3$ correspond to the rocks with higher remanent magnetization, minimum values are correlated with weakly magnetic rocks. The magnetization of samples with the negative inclination is marked minus ( - ) and negative values are given below the $\mathrm{x}$-axis. They correspond to the geomagnetic field with reversed polarity. 
the present field intensity. The same value is evident during a short reversal to normal polarity, but after returning to reversed polarity, in its final phase, the field acquired nearly three times its present intensity. After reversal to normal polarity, the intensity is very low, i.e. 0.2 to 0.7 times the present intensity. In the period of rapidly alternating positive and negative polarities, which represents a certain intermediate phase, the intensity was very low again and only rarely exceeded the value of $0.475 \mathrm{Oe}$, which corresponds to present-day field. Afterwards values with normal polarity only followed and a large increase of geomagnetic intensity occurred when the field achieved maximum values of the triple present-day field intensity. As a whole we may distinguish very expressive dynamic changes in the variation of the geomagnetic field at certain periods and a very calm field at others. The geomagnetic intensity after reversal and mainly in the intermediate phase is very low. This phenomen substantiates some theoretical conclusions concerning the total disappearance of the main geomagnetic field during the process of reversal and its successive increase under reversed polarity. The field, investigated in the intermediate phase, in which it acquires a value of $0.2 \mathrm{Oe}$, corresponds to the nondipole field which probably only existed at the time of the reversal. The changes in the pole positions during the process of reversal are given in Fig. 11, the values of the geomagnetic intensity for the individual poles being given in Oe. It appears that the smaller the intensity of the earth's magnetic field, the closer to the equator are the pole positions. The tendency of the westward polar wandering is remarkable; the similarity of the character of the earth's magnetic field during reversal to that of the non-dipole field is thus proved especially as regards the westward drift. The results acquired provide now experimental data for the development of theories dealing with the geomagnetic reversal.

\section{Earth's magnetic field changes in Quaternary and Polarity events}

Eight sedimentary cycles which occurred during the Quaternary were dated astronomically by means of the winter insolation curve; thus, they offer a possibility for investigating the earth's magnetic field during the past one milion years. Besides cycles $I, H$, during the sedimentation of which the field reversal took place (Fig. 7, 8), we also examined cycles $G$ to $B$ at the localities of Cerveny kopec near Brno and Sedlec near Prague (Figs. 12 to 17). As the age, at which the sedimentation of the individual cycles commenced, is known approximately, we took around 50 samples (cubes with the edge of $2 \mathrm{~cm}$ ) from the base of every cycle per one meter of thickness, which represents approximately $5-10,000$ years of sedimentation. This means, e.g. for cycle $G$ that the magnetic changes shown in Fig. 12 ab cover a period of about 726,000-716,000 years. Every sample in this case represents the age of 100-200 years. A similar method of magnetic investigation to that used for examining the geomagnetic reversal was applied. Contrary to the previous data, magnetic cleaning identified only a very small unstable magnetization here which could not influence the stable remanent magnetization by more than $2 \%$; therefore, we could construct the curves characterizing changes of $D$ and $I$ for the individual cycles.

The samples of cycle $G$ (approximate age 726,000 years-Fig. 12ab) and of cycle $F$ (age 


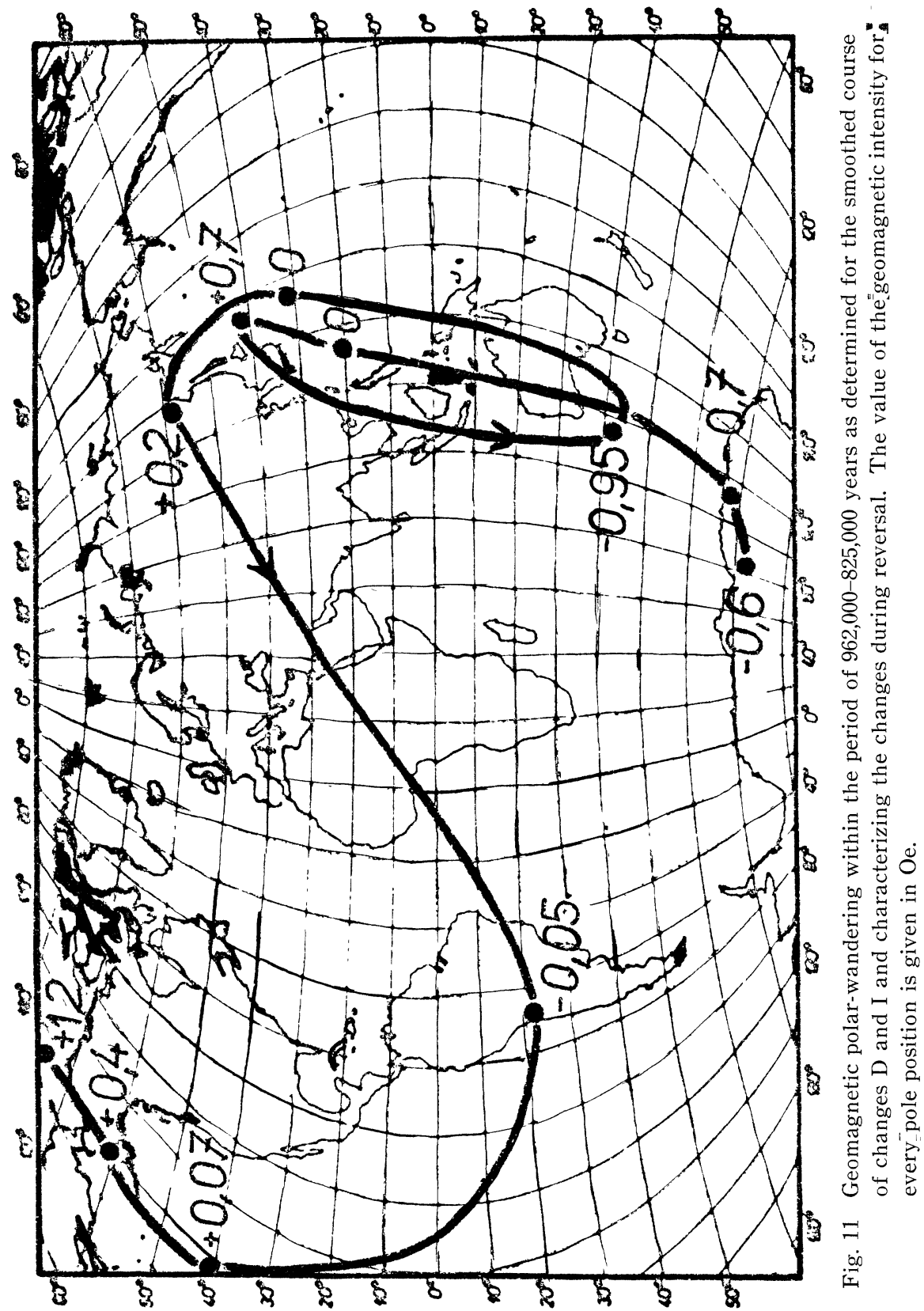

602,000 years-Fig. $13 \mathrm{ab}$ ) show a relatively calm course of the field, mainly in inclination, which is close to its present value. In cycle $E$ (age 485,000 years) much greater changes in declination were found (Fig. $14 \mathrm{a}$ ) and the inclination showed some negative values (Fig. 14b). In order to verify the validity of these short reversals other samples from the same cycle were taken at a distance of $100 \mathrm{~m}$ from the place where cycle $E$ was investigated at first. It appeared that he changes of the field are of similar character and also 


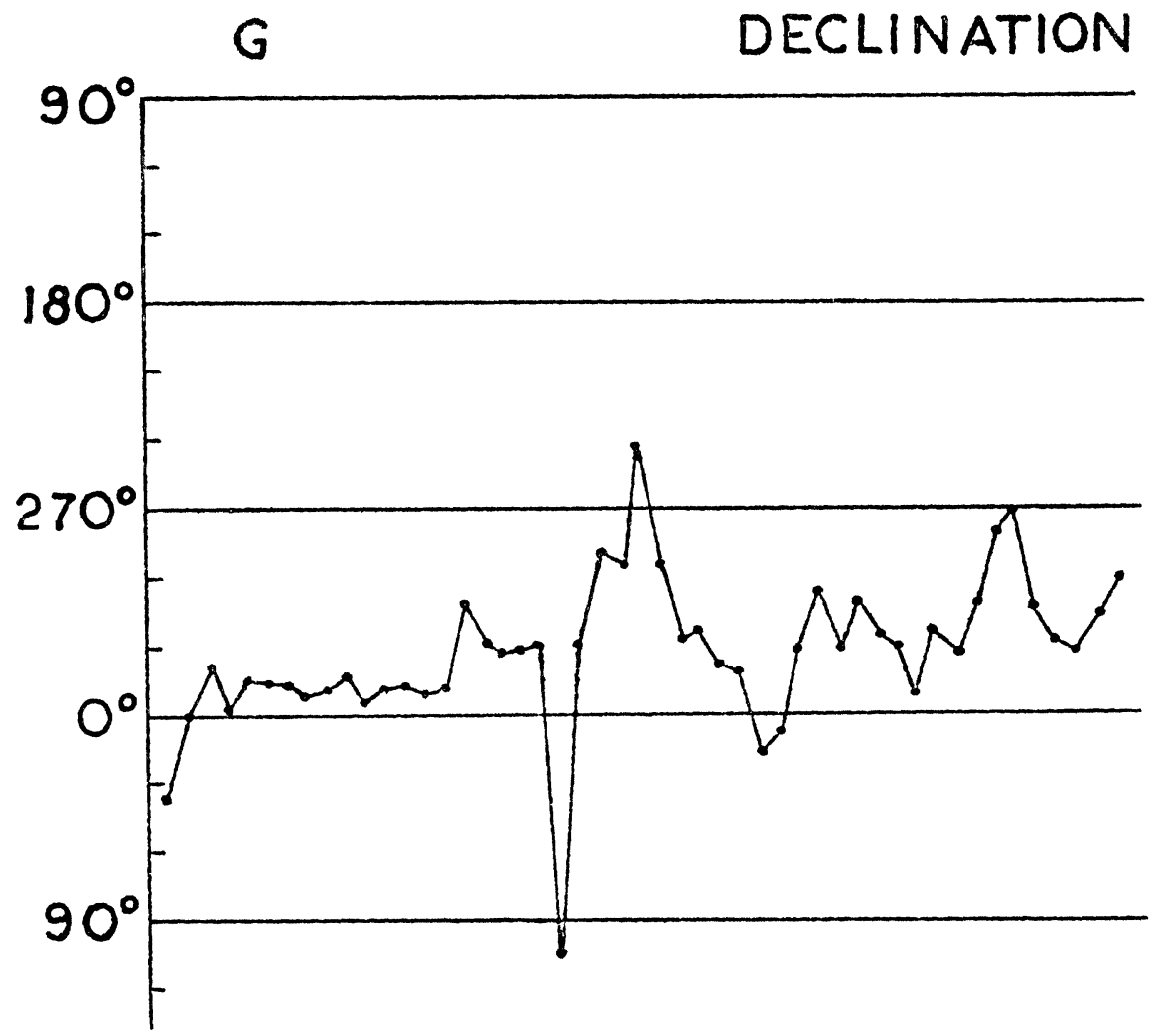

Fig. 12a Temporal dependence of changes in magnetic declination for sedimentary cycle $\mathrm{G}(726,000$ years). The curve represents the changes for time interval of about 10,000 years.
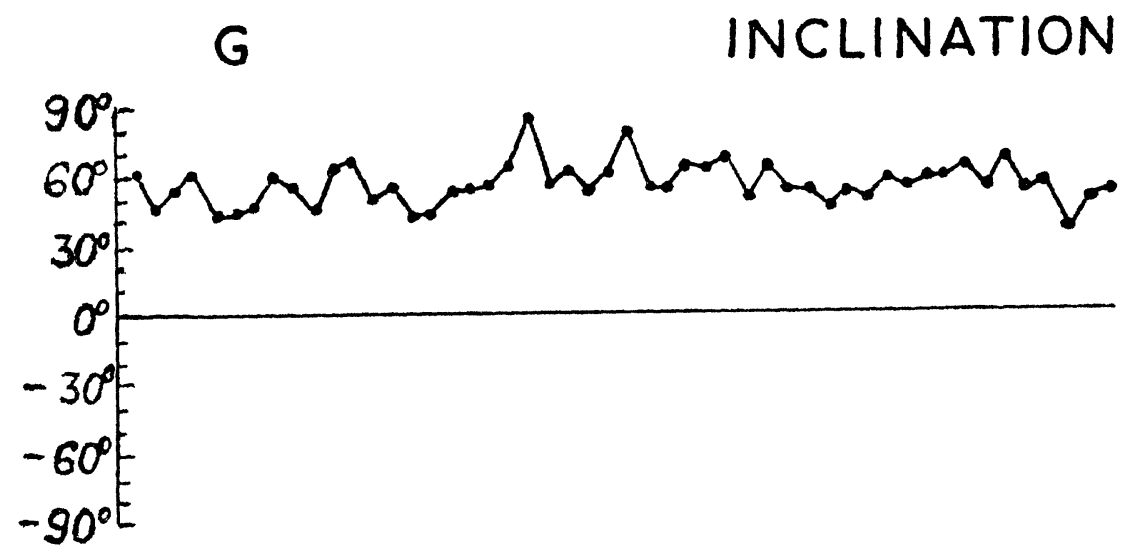

Fig. 12b Temporal dependence of changes in magnetic inclination for sedimentary cycle $\mathrm{G}(726,000$ years)

short events with reversed polarity were found to be the same (compare Fig. 14b with Fig. 14c).

The remarkable conclusion, issuing from the results obtained in Figs. 15 to 17 concerns 


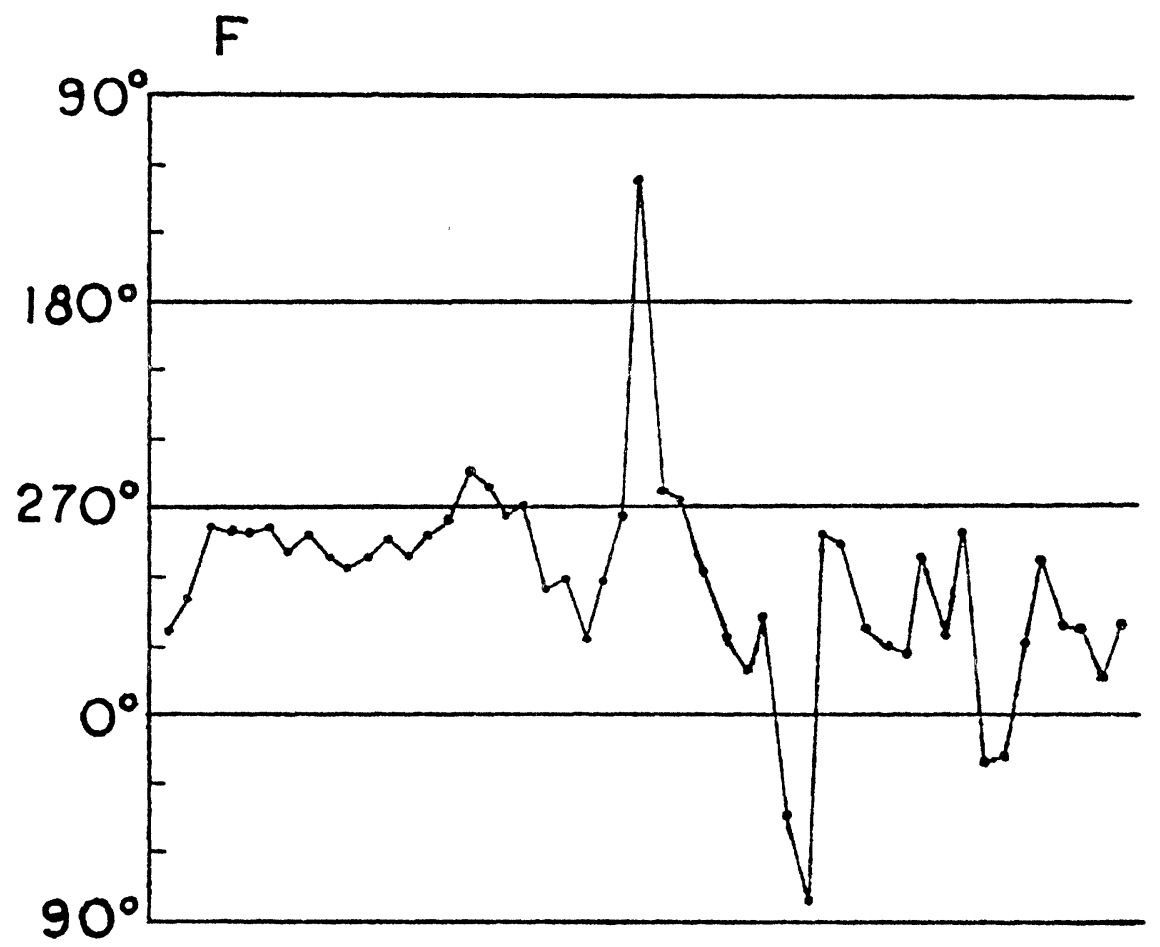

Fig. 13a Magnetic declination for cycle F (602,000 years)

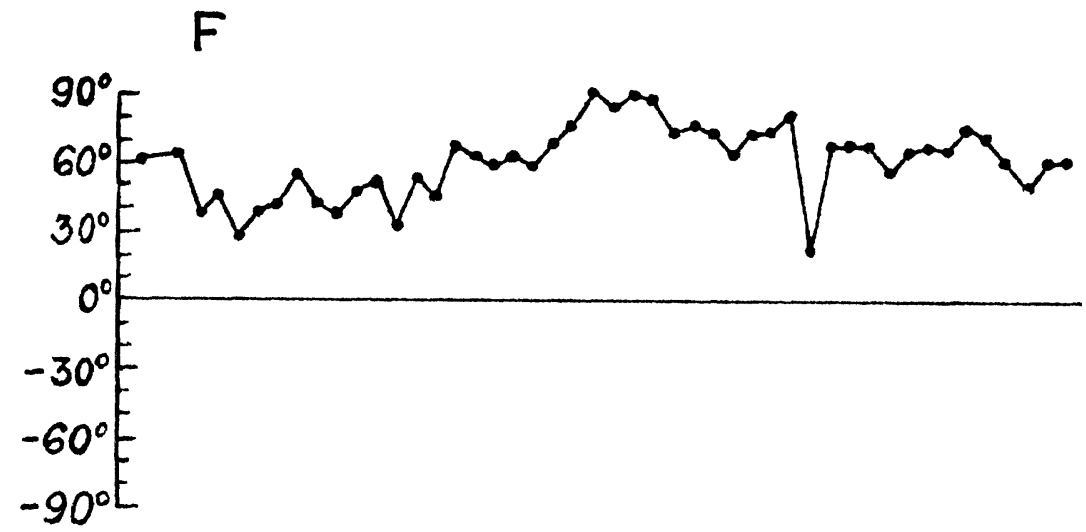

Fig. 13b Magnetic inclination for cycle $\mathrm{F}(602,000$ years $)$

the occurrence of negative $I$ and $D$ values, amounting to about $180^{\circ}$ for cycles $D$ (age 370,000 years), $C$ (243,000 years), as well as at $B$ (128,000 years). Although until recently it was assumed that the period with reversed polarity occurred approximately over 900,000 years ago, our results show that the field changes, even in younger periods, were very dynamic, so that in some cases short temporal reversals could have occurred. This is in agreement with Cox (1968), who suggested a geomagnetic reversal model, predicting short reversals of polarity. For the majority, however, the directions of remanent magnetization 


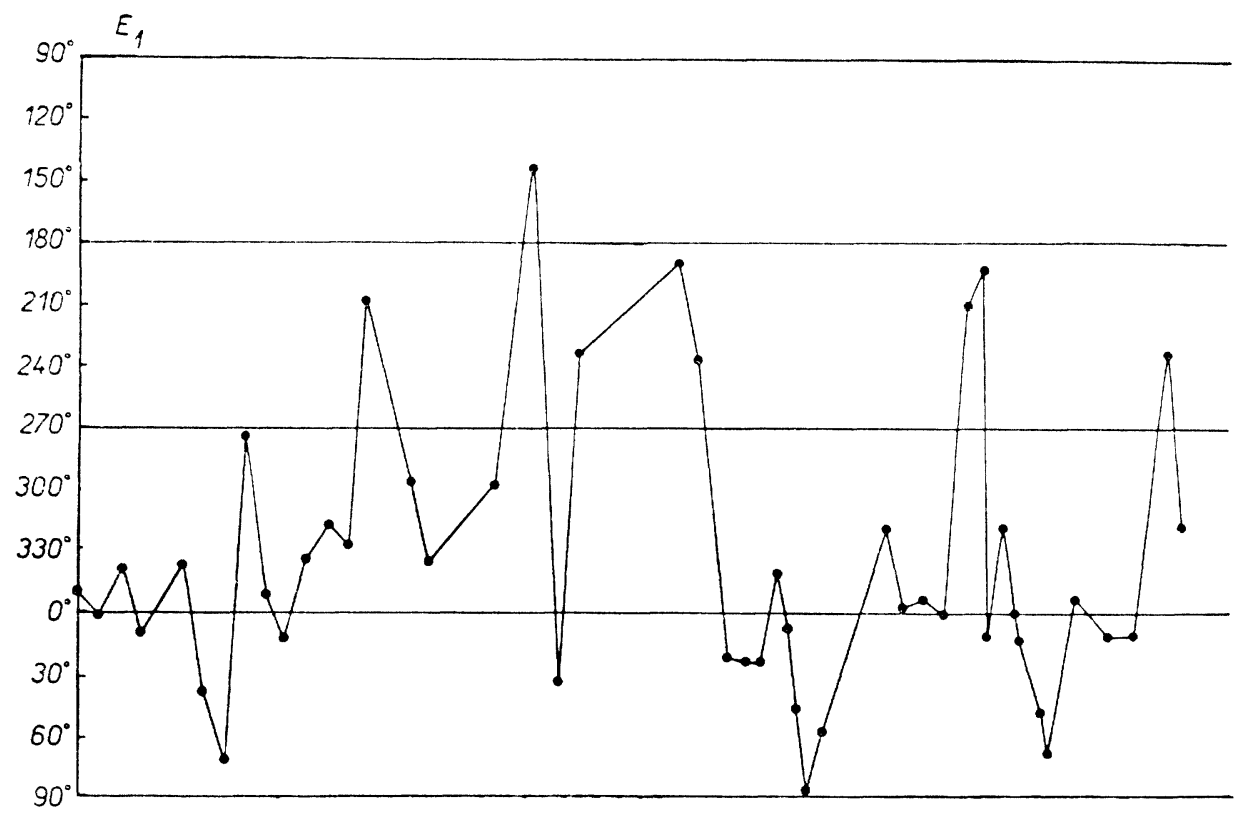

Fig. 14a Magnetic declination for cycle $\mathrm{E}$ (485,000 years)

\section{$E_{1}$}

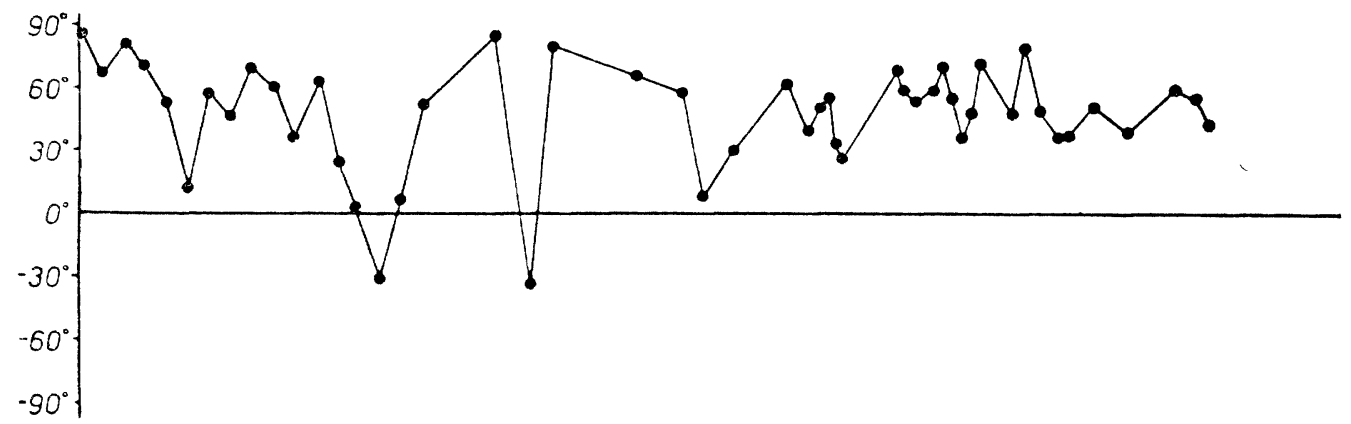

Fig. 14b Magnetic inclination for cycle E (485,000 years)

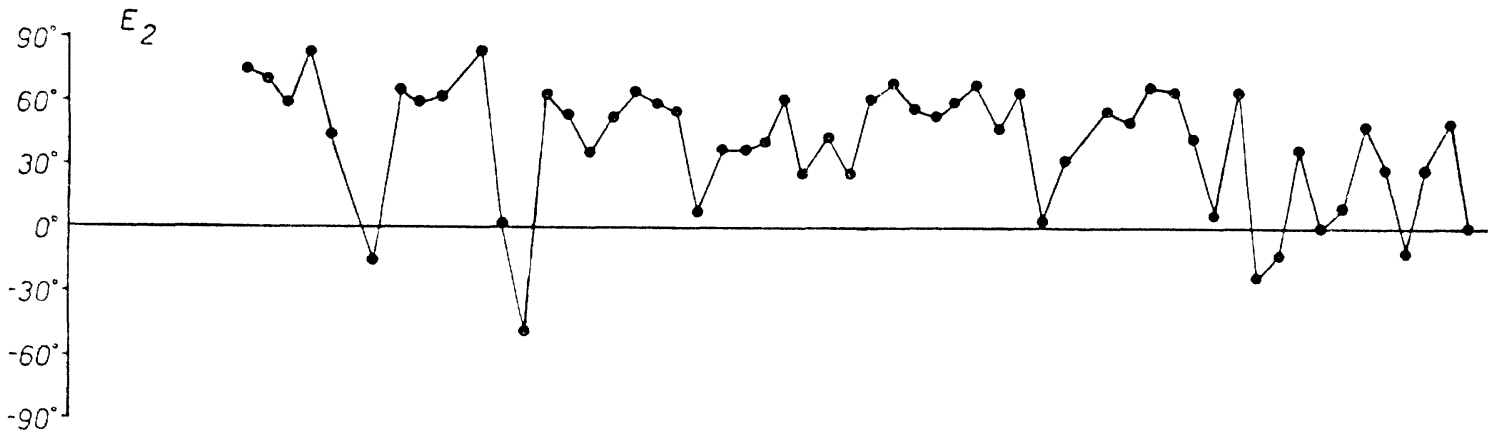

\section{$14 \mathrm{c}$}

Fig. 14c Magnetic inclination for cycle E, samples collected at a distance of $100 \mathrm{~m}$ from the place where cycle $\mathrm{E}$ was investigated at first (compare with Fig. 14b) 


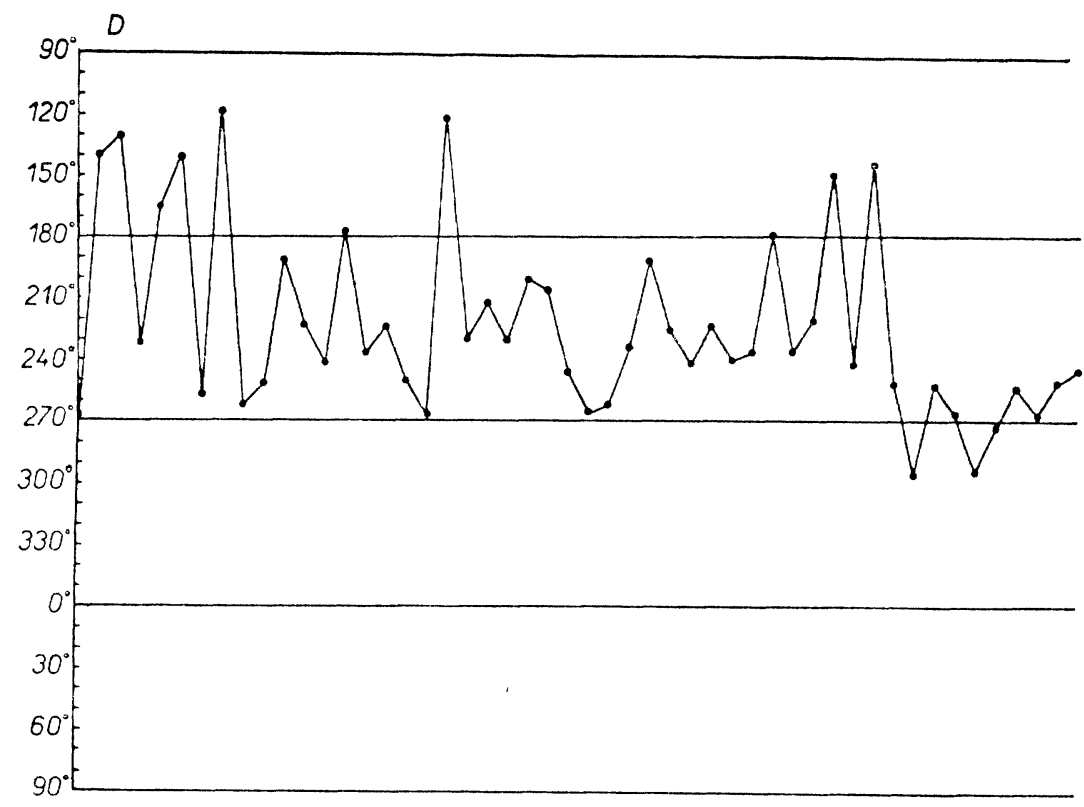

Fig. 15a Magnetic declination for cycle D (370,000 years)

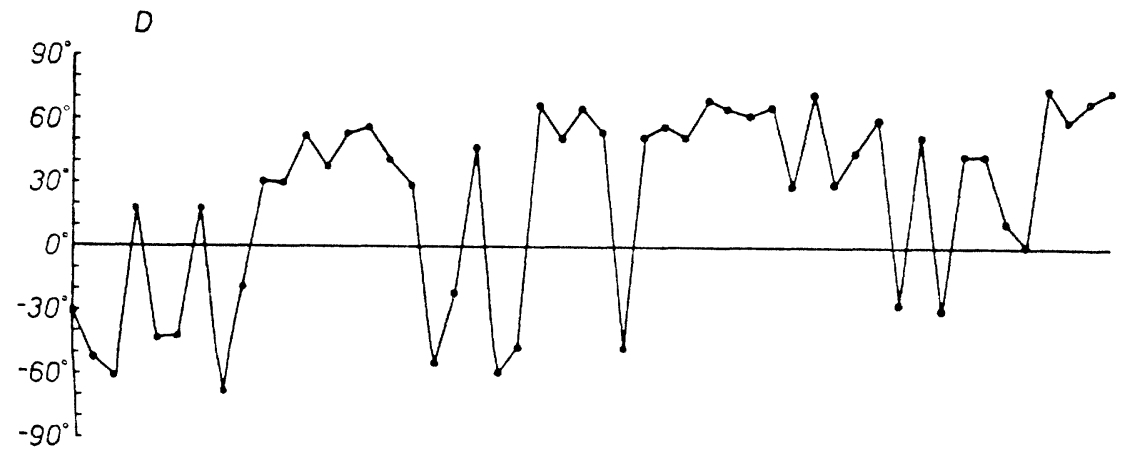

Fig. 15b Magnetic inclination for cycle D (370,000 years)

close to the present-day geomagnetic field prevail $\left(I=60^{\circ}\right)$. When we consider that every $2 \mathrm{~cm}$ cube sample represents a time interval of around 200 years then it is clear that the curves in Figs 12-17 only represent the main features of the earth's magnetic field. The relatively big oscillations for same time intervals and the frequent but only short-termed occurrence of reversed values, hitherto only assumed, are surprising.

On comparing the declination for sedimentary cycles $D, C$ and $B$, we can see that in cycle $D$ (around 340,000 years) smaller oscillations between $160^{\circ}$ and $270^{\circ}$ prevail; the mean value amounts to $215^{\circ}$ (Fig. 15 a) (Bucha, Horacek, Koci, in press). On the other hand, sedimentarly cycle $C(243,000$ years) shows considerably higher oscillations practically for the whole diapason of declination between $9^{\circ}$ and $360^{\circ}$ (Fig. 16a). Cycle $B$ (128,000 years) displays oscillations between $230^{\circ}$ and $350^{\circ}$, the mean value being around $300^{\circ}$ (Fig. 17a). A similar character of the changes was found for profiles of the same age from other 


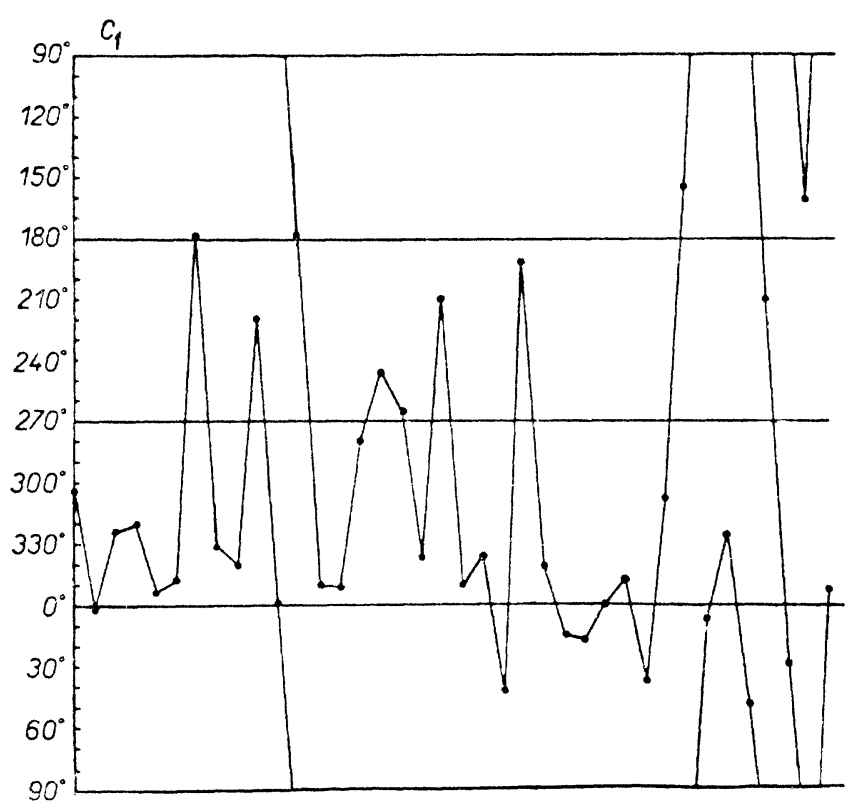

Fig. 16a Magnetic declination for cycle C (243,000 years)

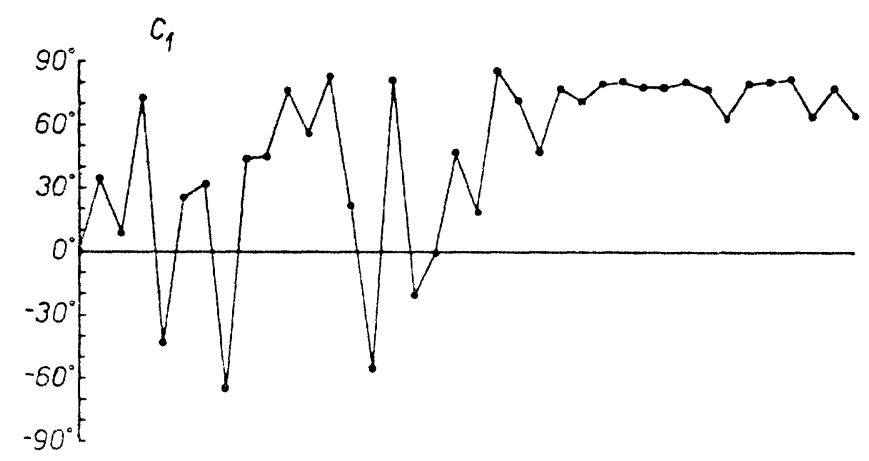

Fig. 16b Magnetic inclination for cycle $\mathrm{C}(243,000$ years $)$

localities. Differences in the changes of $I$ appear in the individual cycles, too. While some expressive reversals took place in cycle $D$ (Fig. 113b), when the negative inclination reached $-60^{\circ}$ (i.e. the total reversal of the polarity manifested), cycle $C$ (Fig. 16b) contains a considerably lower number of short-term reversals in the upper half of the profile, only. Finally, in the case of cycle $B$ (Fig. 17b), negative directions of $I$ were only ascertained for isolated samples, the inclination displaying low negative values; this means that only partial oscillations took place, not total reversals.

The configuration of changes in declination and inclination within the different cycles provides a possibility of elaborating new palaeomagnetic correlation dating methods.

I thank J. Horacek, A. Koci and V. Cerny for competent experimental assistance. 


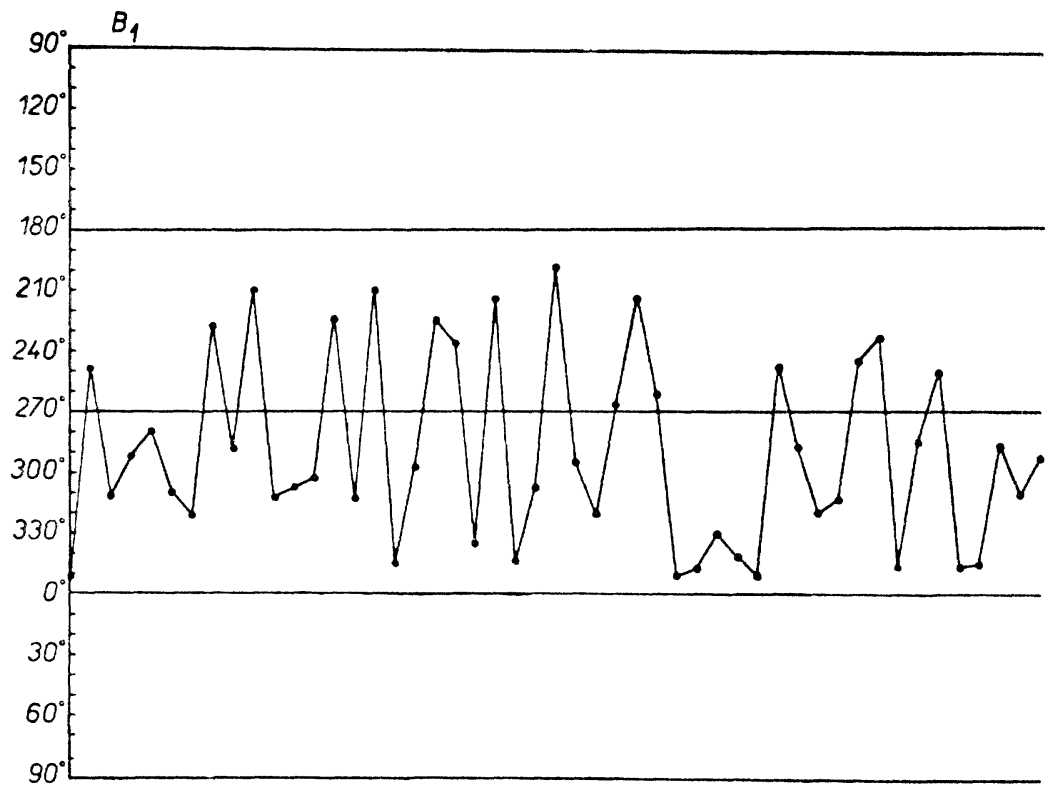

Fig. 17a Magnetic declination for cycle B (128,000 years)

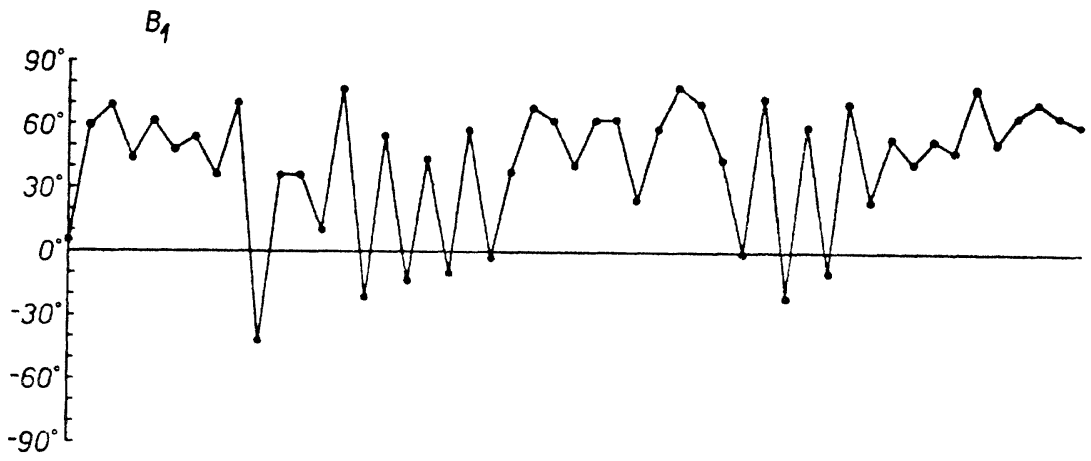

Fig. 17b Magnetic inclination for cycle B (128,000 years)

\section{References}

Bucha V., Intensity of the earth's magnetic field during archaeological times in Czechoslovakia. Archaeometry 10, 12-22, 1967.

Bucha V., Horácek J., Koci A., Kukla J., Palaomagnetische Messungen in Lòss. Periglaziazone, Loss und Paleolithicum in Tschechoslowakei, Brno (in press).

Cox A., Lengths of geomagnetic polarity reversals. J. Geophys. Res. 73, No. 10, 3247-3260, 1968.

Jelinek V., A high sensitivity spinner magnetometer. Studia geoph. et geod. 10, 58-78, 1966.

Kukla J., Dating Pleistocene by balance of obtained solar heat. Vestnik UUG, XLIII, 215, 1968. 